\title{
Avaliação de desempenho dos sistemas de abastecimento de água do Agreste brasileiro
}

\author{
Performance measurement of the water distribution \\ systems of the Brazilian Agreste region
}

\section{Saulo de Tarso Marques Bezerra \\ Monica Pertel J osé Eloim Silva de Macêdo}

\section{Resumo}

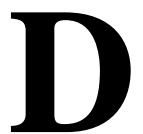

e acordo com os dados do Sistema Nacional de Informações sobre Saneamento (SNIS) do Brasil, do total da água produzida pelas companhias de saneamento em 2016, cerca de 38\% foram perdidos na distribuição, entre perdas reais e aparentes. Além das perdas de água, perdem-se outros insumos, recursos financeiros e mão de obra que poderiam ser investidos na melhoria e na reabilitação de ativos das empresas. A presente pesquisa objetiva avaliar o abastecimento de água de municípios do Agreste brasileiro com base em indicadores do SNIS relacionados às perdas de água. A metodologia fundamentou-se em estatística descritiva, de modo que os valores dos indicadores operacionais adotados de cada munícipio foram comparados com valores de referência, a fim de analisar a eficiência dos sistemas de abastecimento de água e estabelecer um benchmarking da região. Os melhores resultados foram da Embasa (Bahia), enquanto o estado com pior desempenho foi o de Sergipe. O estudo fornece subsídios aos gestores de companhias estaduais para a priorização de investimentos em sistemas ineficientes.

Palavras-chave: Sistema de distribuição de água. Indicadores de desempenho. Perdas de água. Benchmarking.

${ }^{1}$ Saulo de Tarso Marques Bezerra ${ }^{1}$ Universidade Federal de Pernambuco Caruaru - PE - Brasil

${ }^{3}$ Monica Pertel ${ }^{3}$ Universidade Federal do Rio de J aneiro Rio de J aneiro - RJ - Brasil

${ }^{4} \mathrm{~J}$ osé Eloim Silva de Macêdo ${ }^{4}$ Centro Universitário Maurício de Nassau Caruaru - PE - Brasil

Recebido em 28/05/18 Aceito em 19/09/18

\section{Abstract}

According to the 2018 report produced by the National Sanitation Information System (hereby referred to by its Brazilian acronym SNIS), $40 \%$ of the total amount of water treated by state water utilities in 2016 was lost during its distribution, either through real losses or due to apparent losses. In addition to water, other types of loss are incurred in, such as financial resources and labour, moreover, the financial resources associated with these losses could otherwise be invested in improvements of the companies' systems. This study aims to evaluate the performance of state water utilities in controlling and reducing water loss in supply systems based on the SNIS indicators in municipalities of the Brazilian Agreste. The methodology was based on descriptive statistics. Therefore, the adopted values of the operational indicators in each municipality were compared with reference values in order to analyse the efficiency of water supply systems and to establish a benchmark for the region. The best results were from Embasa (Bahia), while the worst performing state was Sergipe. The study provides useful information to managers of state companies when prioritising investments to improve inefficient systems.

Keywords: Water supply system. Performance indicators. Water losses. Benchmarking. 


\section{Introdução}

A água e a sua governança constam como um dos capítulos centrais na agenda internacional nas últimas décadas, sendo reconhecida pela sociedade como um recurso limitado, escasso e vulnerável. O crescimento populacional, a escalada da demanda, a poluição dos mananciais e os efeitos das mudanças climáticas têm impulsionado reflexões sobre a vulnerabilidade e a natureza finita dos recursos hídricos.

A disponibilidade de água para o abastecimento da população urbana, que durante muito tempo em diversos locais se mostrava suficiente - ou seja, os mananciais nas cercanias das cidades correspondiam ou excediam à demanda -, tornouse escassa em muitos desses locais, resultando na busca por mananciais cada vez mais distantes dos centros urbanos (BARRETO, 2008). Diante disto, é imperativo que os gestores públicos e privados avaliem a eficiência e eficácia dos seus sistemas, gerenciem seus ativos e ponderem antes de adotarem soluções que abordem exclusivamente a ampliação da infraestrutura de transporte e tratamento de água. A promoção de melhorias de desempenho nos ativos de forma geral é essencial para garantir sustentabilidade e disponibilidade de água em longo prazo. É nessa dimensão que Morsch, Mascaró e Pandolfo (2017) ressaltam que os problemas da atualidade necessitam de uma visão mais humana e ecológica, encontrando assim a relação das infraestruturas com o ambiente construído.

De acordo com o Diagnóstico dos Serviços de Água e Esgotos do SNIS para o ano de 2016, o Brasil apresentou um índice médio de perdas de água na distribuição de 38,1\%. Essas perdas são compostas de perdas reais e aparentes. As perdas reais são devido a diversos tipos de vazamentos nos sistemas de abastecimento de água (SAA), enquanto as aparentes originam-se de ligações clandestinas e hidrômetros defeituosos ou fraudados (ALEGRE et al., 2005). As perdas, sobretudo as reais, devem-se principalmente às péssimas condições das instalações dos SAA. A gestão patrimonial da infraestrutura deve ser proativa, com a priorização dos ativos sendo bem avaliada e a reabilitação assumindo um papel central nas decisões.

A rede de ativos gerenciada em SAA sofre com o fato de que parte da infraestrutura dos sistemas está enterrada, de difícil acesso para manutenção e inspeção. Com o objetivo de reverter essa situação comum em diversos países, tem-se adotado o conceito de Asset Management, que é entendido como a gestão estratégica e sustentável do patrimônio. No caso do abastecimento de água, o Asset Management incide sobre a gestão dos ativos fixos tangíveis que compõem os sistemas diretamente associados à prestação do serviço, a saber: as redes de distribuição, os reservatórios, as estações elevatórias e as estações de tratamento. Com o intuito de padronizar e difundir o entendimento sobre a Gestão Patrimonial de Infraestrutura, o Laboratório Nacional de Engenharia Civil (LNEC), juntamente com a Universidade de Lisboa e a Entidade Reguladora dos Serviços de Água e Resíduos de Portugal, publicou dois guias técnicos centrados na reabilitação de SAA e redes de esgotamento sanitário (ALEGRE; COVAS, 2010).

Nesse contexto, o uso de sistemas de indicadores de desempenho é imprescindível para avaliar os SAA. A quantificação dos indicadores é primordial para o refinamento da gestão e pode alavancar sobremaneira os processos de tomada de decisão. Uma vez que permitem a identificação de problemas e a consequente promoção das medidas corretivas, além de apoiarem à formulação de ações, políticas e estratégias mais eficientes. Em suma, são capazes de possibilitar uma visão abrangente, de forma a se obter avaliações consistentes sobre o desempenho dos diversos serviços.

A avaliação de desempenho permite medir as metas e os objetivos da prestação de serviços e se apresenta como a melhor alternativa para o acompanhamento dos serviços prestados, permitindo antever problemas e agilizar a tomada de decisão, seja para medidas estruturais ou estruturantes (GANJIDOOST et al., 2018), com aplicações diretas para os gestores, as agências reguladoras, os investidores, os usuários, os próprios prestadores de serviços e a sociedade. Contudo, a necessidade de implementar sistemas de medição de desempenho na indústria da água, embora tecnicamente indiscutível, ainda encontra barreiras de natureza predominantemente política nos países em desenvolvimento, uma vez que os indicadores cumprem o primordial papel de mostrar como caminha a gestão e as decisões tomadas (VILANOVA; MAGALHÃES FILHO; BALESTIERI, 2015).

O cálculo dos indicadores na mediação de desempenho é tipicamente realizado pela razão entre duas variáveis, podendo ser adimensionais, expressos por meio de percentagem ou intensivos como L/lig./dia (ALEGRE et al., 2006). Ainda segundo os autores, um indicador analisado individualmente pode ter pouco interesse prático. Em contrapartida, o uso de um sistema de indicadores predefinido pode ser adotado para estabelecer um benchmarking. O processo de benchmarking na indústria da água é atualmente um 
tema de importância internacional. Por se tratar de um recurso escasso, o tema é estratégico para governos e reguladores em diversos países nos quais os serviços são monopólios - como Brasil, Chile, Holanda, Inglaterra e Portugal (MOLINOSSENANTE et al., 2016).

A International Water Association (IWA) destacase no âmbito internacional pela definição de um grupo de indicadores de desempenho, estruturados de forma a satisfazer as necessidades comuns dos principais tipos de empresas de saneamento. Para isso foi criado um grupo de trabalho formado por técnicos de companhias e agências reguladoras de sistemas de abastecimento de água de diversos países e, em 2000, foi lançado o primeiro manual de indicadores de desempenho da associação - Best Practice Water Balance and Performance Indicators for Water Supply Services, cujos principais objetivos foram (ALEGRE et al., 2004):

(a) fornecer um quadro de referência de indicadores que constituíssem um instrumento de apoio à gestão das prestadoras de serviço de abastecimento de água;

(b) permitir futuras comparações entre prestadoras no âmbito de iniciativas de benchmarking métrico;

(c) disponibilizar subconjuntos de indicadores para uso de agências reguladoras, de acordo com as suas necessidades específicas; e

(d) fornecer uma base de dados, tendo em vista um alargamento progressivo e a consolidação de estatísticas no âmbito internacional.

O manual possibilita testes e comparações entre diferentes operadores em diversas regiões e países, visto que oferece uma metodologia acessível para as prestadoras (MOLINARI, 2006). Assim, com base nos indicadores da IWA, diversos prestadores no mundo conseguem consolidar seus indicadores e unificar seus resultados, possibilitando a comparação dos sistemas. Esse fato permite a determinação de desempenho e a busca por benchmarkings.

Como exemplo do exposto pode ser observada a boa prática da Asociación de Entes Reguladores de Agua Potable y Saneamiento de las Américas (ADERASA), que foi criada em 2001 por oito países fundadores e, em 2006, já possuía 16 países integrantes. Numa tentativa de facilitar o intercâmbio de informações e identificar as melhores práticas entre as companhias associadas, desde o ano de 2003 desenvolve e utiliza um programa de benchmarking no qual compara as informações de todas as companhias reguladas. Nesse mesmo ano, foi proposto o primeiro manual com indicadores de desempenho baseados nos sistemas da International Benchmarking Network for Water and Sanitation Utilities (IWA e IBNET), para assegurar que os resultados pudessem ser utilizados e comparados com resultados de qualquer outra parte do mundo (SILVA, 2006). A IBNET é uma iniciativa do Banco Mundial com apoio financeiro do Department for International Development do Reino Unido com o objetivo de reunir informações sobre prestadores de serviço de abastecimento de água e esgotamento sanitário de vários países.

Outro exemplo de órgão que adota indicadores para a gestão dos SAA é o Office of Water Services (OFWAT), órgão regulador, econômico e ambiental dos serviços de água e esgotamento sanitário da Inglaterra, País de Gales e de alguns operadores da Austrália e Nova Zelândia, que também utiliza o sistema de indicadores para a gestão dos SAA. O sistema de indicadores da OFWAT é organizado em quatro categorias - serviço ao consumidor, distribuição de água, impactos ambientais e financeiros -, admitindo a verificação do nível de satisfação do consumidor para cada categoria ou de modo global (OFFICE..., 2011).

Nos países escandinavos destaca-se o Six-Cities Group, formado por seis cidades. O grupo iniciouse em 1994, composto por Oslo, na Noruega; Gothenburg, Malmo e Estocolmo, na Suécia; Copenhagen, na Dinamarca; e por Helsinki, na Finlândia. As referidas cidades têm em comum o controle público sobre os serviços de água e esgoto. Os seus gestores optaram por implementar um sistema de benchmarking entre as entidades gestoras com a finalidade de demonstrar a eficiência dos sistemas com a gestão pública.

No Brasil, o SNIS é considerado a mais completa e abrangente base de dados sobre os serviços de saneamento no país. Trata-se de um sistema informatizado que disponibiliza informações dos municípios e dos estados em uma série histórica de 22 anos, cuja última atualização ocorreu no anobase de 2016. No entanto, Britto (2011) ressalta que os indicadores de perdas de água do SNIS são compostos da soma das perdas reais (físicas) e aparentes (não físicas), já que no Brasil, com raras exceções, os prestadores de serviços não mensuram esses dois componentes separadamente. Esse fato, na maioria dos casos, inviabiliza a comparação de pesquisas que adotam dados do SNIS com pesquisas internacionais fundamentadas em indicadores da IWA.

Decorrentes da importância do tema, diversos estudos destacam-se sobre a avaliação do uso de indicadores, desempenho de sistemas e da verificação de benchmarkings, a saber: MolinosSenante et al. (2016) enfatizaram a importância do 
benchmarking para a regulação da prestação de serviços de abastecimento de água. Para tanto, os pesquisadores propuseram um sistema de avaliação de sustentabilidade por meio da agregação de um conjunto de indicadores em uma única medida geral de sustentabilidade. Adotando como referência os indicadores operacionais do SNIS relacionados às perdas de água, Pertel, Azevedo e Volschan Junior (2016) definiram critérios para a avaliação do desempenho operacional das 22 prestadoras regionais (companhias estaduais) do Brasil e, por meio de comparação de quatro indicadores, estabeleceram um benchmarking.

Haider, Sadiq e Tesfamariam (2015) desenvolveram um modelo de benchmarking de desempenho que considerou 47 indicadores de desempenho. O desempenho foi estabelecido pela agregação dos indicadores através do algoritmo TOPSIS (do inglês, Technique for Order Preference by Similarity to Ideal Solution). A metodologia foi empregada em três empresas de médio porte localizadas em British Columbia, Canadá. Bereskie et al. (2017) propuseram uma estrutura hierárquica de benchmarking para indicadores de desempenho de qualidade de água para avaliar SAA de pequeno porte por meio da comparação cruzada entre sistemas similares. A estrutura proposta foi projetada para quantificar as consequências associadas a problemas sazonais e específicos de qualidade da água. Ganjidoost et al. (2018) empregaram indicadores de desempenho de benchmarking, adotados frequentemente pela indústria da água americana, para a avaliação de três SAA localizados em Ontário, Canadá.

Diante da importância do tema, esta pesquisa objetivou analisar o abastecimento de água de municípios do Agreste brasileiro, cuja escassez do recurso é latente, com base em quatro indicadores de desempenho operacionais relacionados às perdas de água em sistemas de abastecimento. A avaliação dos serviços prestados fornece subsídios aos gestores, no sentido da indução de políticas que permitam a adoção de medidas para o controle de perdas com a priorização de investimentos nos sistemas ineficientes, bem como propicia a definição de um benchmarking para a região.

\section{Materiais e métodos}

\section{Área de estudo}

O Agreste brasileiro, uma mesorregião do Nordeste, corresponde a uma área de transição entre a Zona da Mata e o Sertão brasileiro (Figura 1). Essa mesorregião situa-se numa estreita faixa, paralela à costa, que possui como principais características: solos profundos (latossolos e argissolos), com relevo extremamente variável, associados a solos rasos (litossolos) e vegetação variável com predominância de vegetação caducifólia.

O clima predominante é o semiárido, sendo uma região menos úmida que a Zona da Mata e menos seca que o Sertão brasileiro. É uma área sujeita a secas, cuja pluviometria é relativamente baixa e muito irregular ao longo do ano. De acordo com dados do Serviço Geológico do Brasil (CPRM), a precipitação média anual varia de $400 \mathrm{~mm}$ a $1.700 \mathrm{~mm}$. Além disso, estudos realizados baseados em relatórios técnicos do Instituto Nacional de Pesquisas Espaciais (INPE) e do Intergovernmental Panelon Climate Change (IPCC) apontam para o aumento da frequência e da intensidade das secas, o que resultará em redução da disponibilidade hídrica (MARENGO, 2007).

Figura 1 - Mapa da região do Agreste brasileiro

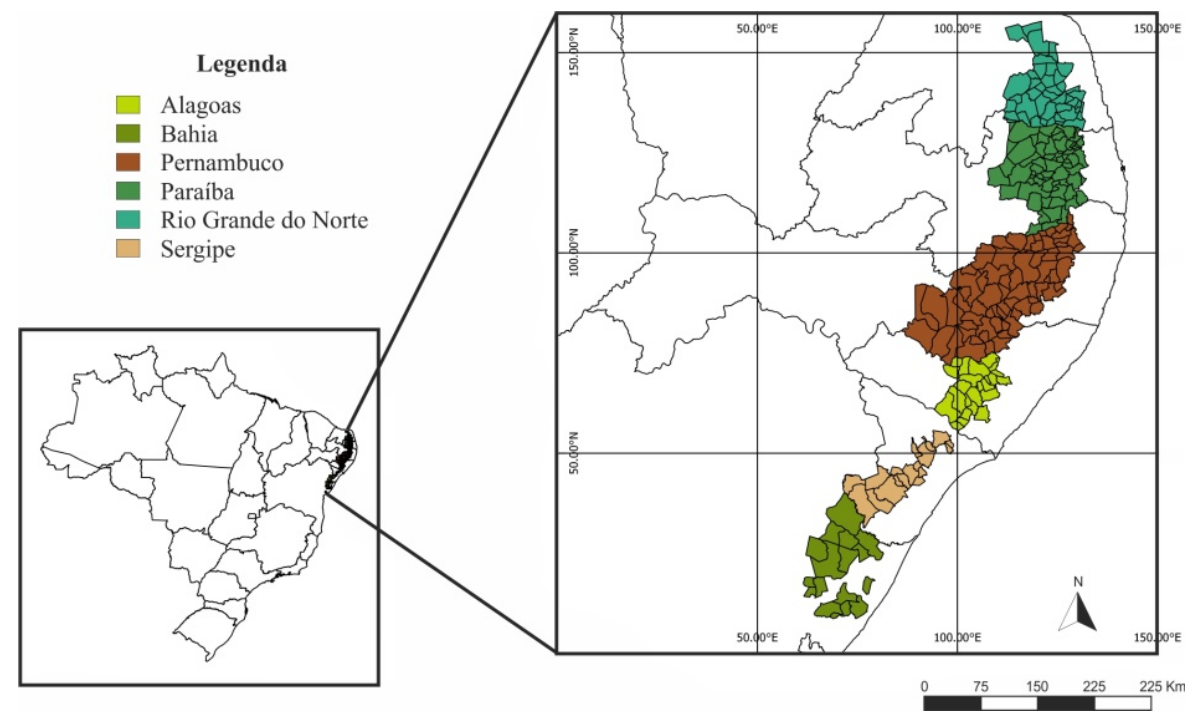


O Agreste possui uma população de aproximadamente 5,3 milhões de habitantes, abrangendo municípios dos estados de Alagoas, Bahia, Paraíba, Pernambuco, Rio Grande do Norte e Sergipe. Por outro lado, das 238 cidades localizadas no Agreste, apenas 13 são abastecidas por companhias locais, as demais são atendidas pelas companhias estaduais, quais sejam: Caern (Rio Grande do Norte [RN]), Cagepa (Paraíba [PB]), Casal (Alagoas [AL]), Compesa (Pernambuco [PE]), Deso (Sergipe [SE]) e Embasa (Bahia [BA]), e estas atendem uma população de 5,06 milhões de habitantes.

\section{Método}

A presente pesquisa definiu o benchmarking dos sistemas de abastecimento de água (SAA) do Agreste brasileiro com base nos valores de referência de indicadores de desempenho disponíveis no SNIS, para o período de 2012 a 2016.

\section{Descrição do banco de dados}

O SNIS é o maior e o mais importante sistema de informações do setor de saneamento do Brasil, com um banco de dados que contém informações de caráter institucional, administrativo, operacional, gerencial, econômico-financeiro e contábil sobre a prestação de serviços de água, de esgotos e de manejo de resíduos sólidos urbanos. A consolidação do SNIS, desde 1995, permite o emprego dos seus indicadores como referência para a avaliação da atuação de empresas e prefeituras. O sistema possui um dispositivo automático de análise de consistência de dados, que alerta os técnicos das companhias para possíveis inconsistências, comparando os dados fornecidos com os benchmarks e o histórico da própria companhia (SISTEMA..., 2018).

As informações do SNIS são processadas pelos técnicos do governo federal, a partir das declarações anuais de responsabilidade das companhias estaduais, empresas e autarquias municipais, empresas privadas e, em muitos casos, prefeituras municipais. Na $21^{\text {a }}$ edição do Diagnóstico dos Serviços de Água e Esgotos (ano-base 2016), o SNIS apurou informações sobre abastecimento de água em 5.172 cidades brasileiras, com população urbana de 170,9 milhões de habitantes, assegurando uma representatividade de $92,9 \%$ em relação ao total de municípios e de 98,1\% em relação à população urbana do Brasil.

No presente estudo, consideraram-se os municípios atendidos pelas companhias regionais, pois essas estão menos propensas a informarem dados arbitrários e são responsáveis pelo abastecimento de $94,5 \%$ do total de municípios da região. Não obstante, a base de dados do SNIS adotada foi municipal.

\section{Indicadores de desempenho avaliados}

Os quatro principais indicadores operacionais disponibilizados pelo SNIS relacionados à avaliação de perdas de água em sistemas de distribuição de água foram adotados (Quadro 1). Os indicadores são expressos pelas Equações 1 a 4.

$I N_{010}=A G 08 \times 100 /$ AGdisp $\quad$ Eq. 1

$I N_{013}=($ AGdisp - AG11 $) \times 100 /$ AGdisp $\quad$ Eq. 2

$I N_{049}=($ AGdisp - AG10 $) \times 100 /$ AGdisp $\quad$ Eq. 3

$I N_{051}=($ AGdisp $-A G 10) /(A G 02 / 0,365) \quad$ Eq. 4

Onde:

AG02 = quantidade de ligações ativas de água;

AG08 = volume anual de água micromedido, em $\mathrm{m}^{3}$;

AG10 = volume anual de água consumido, $\mathrm{em} \mathrm{m}^{3}$;

AG11 = volume anual de água faturado, $\mathrm{em} \mathrm{m}^{3}$;

AGdisp = volume anual de água disponibilizado para distribuição, em $\mathrm{m}^{3}$.

\section{Quadro 1 - Indicadores operacionais adotados nesta pesquisa}

\begin{tabular}{|l|c|c|l|}
\hline \multicolumn{1}{|c|}{ Indicador de desempenho } & Abreviatura & Unidade & \multicolumn{1}{|c|}{ Descrição } \\
\hline Micromedição & $I N_{010}$ & $\%$ & $\begin{array}{l}\text { Volume micromedido em relação ao } \\
\text { volume disponibilizado }\end{array}$ \\
\hline Perdas de faturamento & $I N_{013}$ & $\%$ & $\begin{array}{l}\text { Percentagem de água não faturada em } \\
\text { relação ao volume total produzido }\end{array}$ \\
\hline Perdas totais na distribuição & $I N_{049}$ & $\%$ & $\begin{array}{l}\text { Percentagem de água não distribuída } \\
\text { ao consumidor em relação ao volume } \\
\text { total produzido }\end{array}$ \\
\hline Perdas totais por ligação & $I N_{051}$ & $\mathrm{L.lig}^{-1} \cdot \mathrm{dia}^{-1}$ & $\begin{array}{l}\text { Volume anual de perdas de água na } \\
\text { distribuição por ligação }\end{array}$ \\
\hline
\end{tabular}




\section{Consolidação dos dados da série histórica}

Os dados da série histórica 2012-2016 foram previamente analisados e submetidos a verificações, a fim de retirar da análise estatística aqueles que são abastecidos por prestadoras de serviços locais, apresentam dados inconsistentes indicadores de perdas igual ou menor que zero, e/ou com informações incompletas (falta de informação em pelo menos um ano). A permanência desses dados resultaria, provavelmente, em prejuízo à interpretação da pesquisa. Assim, a metodologia proposta foi aplicada em 186 municípios distribuídos conforme a Tabela 1.

\section{Análise estatística dos dados}

A metodologia adotada para avaliação dos SAA baseou-se em estatística descritiva para a determinação do benchmarking. Os indicadores dos sistemas municipais foram comparados com valores de referência, com intuito de avaliar a eficiência destes e estabelecer os seguintes grupos:

(a) Grupo I - Municípios com melhor desempenho (benchmarking): são aqueles cujo SAA apresenta desempenho superior ao estabelecido como referência, simultaneamente, nos quatro indicadores selecionados; e

(b) Grupo II - Municípios com desempenho médio e baixo: são aqueles cujo SAA não apresenta um bom desempenho em pelo menos um dos indicadores.

Seguindo os mesmos critérios de desempenho estabelecidos por Pertel, Azevedo e Volschan Junior (2015), os valores de referência foram estabelecidos com base no cálculo dos quartis Q1 e Q3 da amostra de dados dos indicadores. Quartis são valores que dividem uma amostra em quatro grupos contendo, aproximadamente, a mesma quantidade de dados. Em estatística descritiva, o primeiro (Q1) e o terceiro (Q3) quartis correspondem aos percentis $p 25$ e $p 75$. Percentis são medidas que dividem a amostra ordenada (por ordem crescente dos dados) em 100 partes, cada uma com uma percentagem de dados aproximadamente igual. O k-ésimo percentil corresponde à frequência cumulativa de $N . k / 100$, onde $N$ é o tamanho amostral. Portanto, os valores de referência foram:

(a) Valor de referência do $I N_{010}=p 75$ da amostra de dados do $\mathrm{IN}_{010}$;

(b) Valor de referência do $I N_{013}=p 25$ da amostra de dados do $I N_{013}$;

(c) Valor de referência do $I N_{049}=p 25$ da amostra de dados do $I N_{049}$; e

(d) Valor de referência do $I N_{051}=p 25$ da amostra de dados do $I N_{051}$.

Os SAA municipais foram enquadrados no Grupo I (benchmarking) quando apresentaram, simultaneamente, indicadores com valores iguais ou melhores que os de referência, ou seja, atendiam as seguintes exigências:

(a) $I N_{010} \geq p 75$ (terceiro quartil); $\mathrm{e}$

(b) $I N_{013} \leq p 25$ (primeiro quartil), $I N_{049} \leq p 25 \mathrm{e}$ $I N_{051} \leq p 25$.

O boxplot foi adotado para analisar a distribuição dos indicadores por classes de população. As linhas inferior e superior da caixa representam, graficamente, Q1 e Q3, enquanto Q2 (mediana) é representado pela linha central. Os gráficos também exibem os limites superior e inferior dos dados "típicos” da amostra avaliada, que são representados pelas linhas em formato " $\mathrm{T}$ ”.

\section{Resultados e discussões}

Baseado nas informações do banco de dados do SNIS, para o período de 2012 a 2016, definiram-se os critérios de desempenho mínimo do Grupo I (benchmarking) da seguinte forma:

(a) índice de micromedição $\left(I N_{010}\right)$ igual ou superior a $57,71 \%$;

(b) índice de perdas no faturamento $\left(I N_{013}\right)$ igual ou inferior a $4,14 \%$;

(c) índice de perdas na distribuição (IN $\left(N_{049}\right)$ igual ou inferior a 26,43\%; e

(d) índice de perdas por ligação $\left(I N_{051}\right)$ igual ou inferior a 114,81 L.lig ${ }^{-1}$.dia ${ }^{-1}$.

Tabela 1 - Municípios avaliados e a população atendida pelas companhias regionais

\begin{tabular}{l|c|c|c}
\hline \multicolumn{1}{c|}{ Estado } & $\begin{array}{c}\text { Companhia } \\
\text { estadual }\end{array}$ & $\begin{array}{c}\text { Quantidade de } \\
\text { municípios }\end{array}$ & $\begin{array}{c}\text { População } \\
\text { (milhares) }\end{array}$ \\
\hline Alagoas & Casal & 17 & 534,6 \\
Bahia & Embasa & 14 & 276,8 \\
Paraíba & Cagepa & 42 & 978,4 \\
Pernambuco & Compesa & 57 & $1.967,2$ \\
Rio Grande do Norte & Caern & 38 & 358,9 \\
Sergipe & Deso & 18 & 446,2 \\
\hline
\end{tabular}


Os resultados consolidados da avaliação são apresentados na Tabela 2, onde se observa a quantidade de municípios, por estado, que obtiveram desempenho superior aos valores de referência nos quatro indicadores. A análise estatística apontou que $10 \%$ dos SAA foram enquadrados no Grupo I - benchmarking.

Os melhores resultados foram da Embasa (BA), com $43 \%$ dos municípios apresentando valores dentro dos limites de referência. As cidades com melhores indicadores foram Itanagra (BA) e Ibirajuba (PE), enquanto as cidades de Quebrangulo (AL) e Bento Fernandes (RN) apresentaram os piores resultados. Apesar de Sergipe apresentar a terceira menor disponibilidade hídrica do Brasil, a Companhia Estadual Deso (SE) obteve o pior desempenho, não possuindo SAA com, pelos menos, um dos quatros indicadores enquadrado dentro dos valores de referência. A disponibilidade do estado é $1.743 \mathrm{~m}^{3}$ /ano para cada habitante (BARTH, 2002), quando a Organização das Nações Unidas estabelece como referência para escassez hídrica o valor de 2.500 m³/ano.

Ressalta-se que os valores negativos de perdas de faturamento ocorrem, provavelmente, porque na região predominam cidades pequenas (população média das cidades com $I N_{013}$ negativo igual a 21.626 habitantes) sujeitas à severa intermitência no abastecimento. Nessas cidades, a maioria da população é de baixa renda e possui um consumo de água inferior a consumação mínima faturada pelas companhias (10 m³/mês).
A Tabela 3 apresenta os valores médios dos indicadores de desempenho dos municípios, por estado. Comparando os valores médios das cidades do Agreste brasileiro com o Nordeste e o Brasil, ano-base 2016 (Tabela 4), constatou-se que os SAA da região têm melhor desempenho que a média nordestina e brasileira. Consequentemente, todas as cidades incluídas no Grupo I obtiveram melhores indicadores que a média brasileira. Para o indicador de micromedição $\left(I N_{010}\right), 51 \%$ dos municípios obtiveram resultados melhores que os do Brasil. Para os indicadores de perdas de faturamento $\left(I N_{013}\right)$, perdas na distribuição $\left(I N_{049}\right)$ e perdas por ligação $\left(I N_{51}\right), 76 \%$, 54\% e 83\% dos municípios obtiveram resultados inferiores à média brasileira, respectivamente.

Os resultados da avaliação dos SAA da região estão apresentados, segundo diferentes estratos populacionais, na Figura 2. O objetivo de tal análise foi inferir eventuais distorções dos resultados anteriores frente à dimensão e à abrangência dos diversos sistemas de abastecimento de água. Os três primeiros estratos populacionais possuem comportamentos semelhantes nos quatro indicadores, enquanto as cidades mais populosas ( $4^{\circ}$ estrato) têm uma concentração de dados em faixas menores e baixa dispersão.

Observa-se nos gráficos da Figura 2 que, com exceção do $I N_{051}$, a distribuição dos dados é aproximadamente simétrica. Logo, é possível inferir que a análise estatística do índice de perdas por ligação possui menor precisão quando comparada aos demais. Além disso, não há relação

Tabela 2 - Quantidade de municípios cujos indicadores atendem aos valores de referência

\begin{tabular}{l|c|c|c}
\hline \multicolumn{1}{c|}{ Estado } & $\begin{array}{c}\text { Companhia } \\
\text { estadual }\end{array}$ & $\begin{array}{c}\text { Quantidade de } \\
\text { municípios }\end{array}$ & $\begin{array}{c}\text { População } \\
\text { (milhares) }\end{array}$ \\
\hline Alagoas & Casal & 17 & 534,6 \\
Bahia & Embasa & 14 & 276,8 \\
Paraíba & Cagepa & 42 & 978,4 \\
Pernambuco & Compesa & 57 & $1.967,2$ \\
Rio Grande do Norte & Caern & 38 & 358,9 \\
Sergipe & Deso & 18 & 446,2 \\
\hline
\end{tabular}

Tabela 3 - Valores médios dos indicadores de desempenho dos municípios, por estado

\begin{tabular}{|c|c|c|c|c|}
\hline $\begin{array}{c}\text { Companhia } \\
\text { Estadual }\end{array}$ & $\begin{array}{c}\text { INo10 } \\
(\%)\end{array}$ & $\begin{array}{c}I N_{013} \\
(\%)\end{array}$ & $\begin{array}{c}\text { IN } 049 \\
(\%)\end{array}$ & $\begin{array}{c}I N_{051} \\
\left(\mathrm{L.lig}^{-1} \cdot \mathrm{dia}^{-1}\right)\end{array}$ \\
\hline Caern (RN) & 36,50 & 43,86 & 40,23 & 473,69 \\
\hline Cagepa (PB) & 64,96 & 5,38 & 24,16 & 107,53 \\
\hline Casal (AL) & 48,00 & 12,31 & 26,88 & 145,32 \\
\hline Compesa (PE) & 52,65 & 11,16 & 41,23 & 242,39 \\
\hline Deso (SE) & 39,32 & 31,62 & 47,02 & 372,96 \\
\hline Embasa (BA) & 45,66 & 40,09 & 54,07 & 375,51 \\
\hline
\end{tabular}


Tabela 4 - Valores médios dos indicadores de desempenho do Agreste segundo diferentes estratos populacionais, série histórica 2012-2016

\begin{tabular}{|c|c|c|c|c|c|}
\hline $\begin{array}{c}\begin{array}{c}\text { Estrato } \\
\text { populacional } \\
\text { (habitantes) }\end{array} \\
\end{array}$ & $\begin{array}{l}\text { Quantidade de } \\
\text { municípios }\end{array}$ & $\begin{array}{l}I N_{010} \\
(\%)\end{array}$ & $\begin{array}{c}I N_{013} \\
(\%)\end{array}$ & $\begin{array}{l}I N_{049} \\
(\%)\end{array}$ & $\begin{array}{c}I N_{051} \\
\left(\mathrm{L.lig}^{-1} \cdot \text { dia }^{-1}\right)\end{array}$ \\
\hline 0 a 10.000 & 60 & 44,32 & 23,00 & 39,03 & 276,50 \\
\hline 10.001 a 20.000 & 60 & 48,79 & 19,75 & 38,02 & 290,43 \\
\hline 20.001 a 50.000 & 49 & 50,25 & 19,72 & 38,74 & 245,06 \\
\hline Mais de 50.000 & 17 & 47,91 & 21,53 & 43,57 & 258,00 \\
\hline 0 a 10.000 & 60 & 48,79 & 19,75 & 38,02 & 290,43 \\
\hline
\end{tabular}

Figura 2 - Comparativo dos indicadores por classes de população

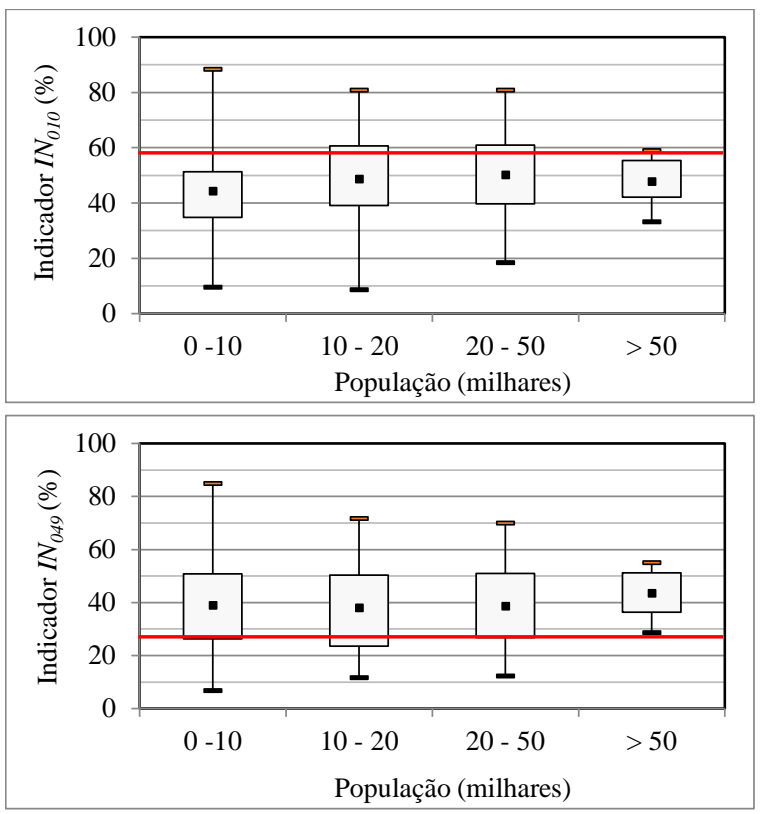

entre a eficiência dos SAA e o número de habitantes dos municípios. Os valores médios dos indicadores não variam, significativamente, segundo os estratos (Tabela 5).

Os valores de referência dos indicadores definidos nesta pesquisa foram mais restritivos que os determinados por Pertel, Azevedo e Volschan Junior (2016). Com base nos valores de referência desses autores, que adotaram dados das companhias estaduais - ano-base 2010 - nos seus cálculos, 19 cidades foram agrupadas como “municípios que atendem ao benchmarking". Avaliando somente os indicadores de perdas de água $\left(I N_{013}, I N_{049}\right.$ e $\left.I N_{051}\right), 66 \mathrm{SAA}$ atenderam aos valores de referência. Os resultados consolidados dessa avaliação estão apresentados na Tabela 5, que também dispõe da quantidade de cidades que atenderam, concomitantemente, aos quatro valores
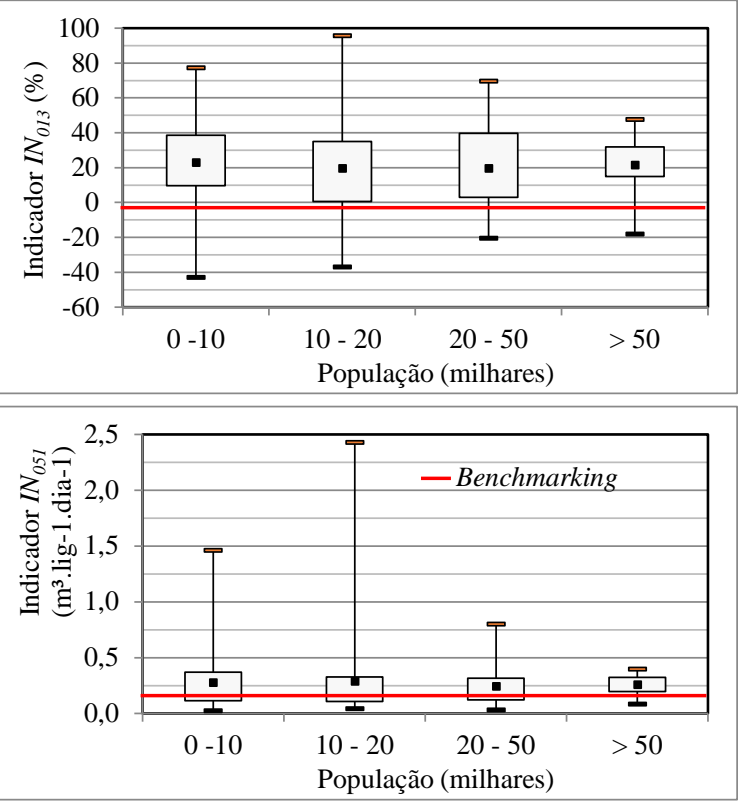

de referência adotados por Pertel, Azevedo e Volschan Junior (2016).

\section{Conclusões}

Esta pesquisa avaliou o fornecimento de água das companhias estaduais de abastecimento de água que atuam no Agreste brasileiro, tendo como base os indicadores de desempenho definidos pelo SNIS relacionados às perdas de água. A avaliação dos serviços prestados fornece subsídios aos gestores para a priorização de investimentos nos sistemas mais ineficientes; propicia às agências reguladoras um estudo para comparação da prestação de serviços nos diversos municípios da região, resultando na definição de um benchmarking local; além de fornecer aos profissionais nordestinos valores de referência para os indicadores determinados em condições sociais, ambientais e econômicas semelhantes às dos seus sistemas. 
Tabela 5 - Quantidade de municípios cujos indicadores atendem aos valores de referência de Pertel, Azevedo e Volschan J unior (2016) - ano-base 2010

\begin{tabular}{|c|c|c|c|c|c|}
\hline \multirow{4}{*}{$\begin{array}{l}\text { Companhia } \\
\text { Estadual }\end{array}$} & $\begin{array}{l}\text { INo10 } \\
(\%)\end{array}$ & $\begin{array}{l}\text { IN } 013 \\
(\%)\end{array}$ & $\begin{array}{l}\text { INo49 } \\
(\%)\end{array}$ & $\begin{array}{c}\text { INo51 } \\
\left(\mathrm{L.lig}^{-1} \cdot \text { dia }^{-1}\right)\end{array}$ & \multirow{4}{*}{$\begin{array}{c}\text { Quantidade } \\
\text { de } \\
\text { municípios } \\
\text { classificados } \\
\text { no Grupo I }\end{array}$} \\
\hline & \multicolumn{4}{|c|}{$\begin{array}{l}\text { Valores de referência para o benchmarking } \\
\text { (percentil 25\%) }\end{array}$} & \\
\hline & 65,48 & 24,10 & 33,00 & 254,20 & \\
\hline & \multicolumn{4}{|c|}{$\begin{array}{c}\text { Quantidade de munícipios com } \\
\text { desempenho superior ao valor de referência }\end{array}$} & \\
\hline Casal (AL) & 1 & 4 & 8 & 6 & 1 \\
\hline Embasa (BA) & 6 & 13 & 13 & 14 & 6 \\
\hline Cagepa (PB) & 4 & 34 & 29 & 38 & 4 \\
\hline Compesa (PE) & 7 & 41 & 15 & 41 & 7 \\
\hline Caern (RN) & 1 & 13 & 7 & 13 & 1 \\
\hline Deso (SE) & - & - & - & - & - \\
\hline Total & 19 & 105 & 72 & 112 & 19 \\
\hline
\end{tabular}

Os melhores resultados foram da Bahia (Embasa), com 43\% dos municípios apresentando performance superior aos limites de referência, enquanto o estado com pior desempenho foi o de Sergipe, que possui a terceira menor disponibilidade hídrica do Brasil. Os dados avaliados ratificam que o acesso aos serviços de água não é determinado somente pela escassez hídrica, embora esse aspecto seja frequentemente citado como o único motivo.

Apesar dos resultados demonstrarem que a maioria dos sistemas de abastecimento de água do Agreste possuir indicadores melhores que a média nordestina e brasileira, destaca-se a necessidade da continuidade dos investimentos em infraestrutura e na gestão eficiente dos sistemas da região.

Como restrição ao uso dos resultados da pesquisa, identificou-se, como uma das limitações do SNIS, a falta de dados de perdas reais e aparentes, o que impossibilita o cálculo de parte dos principais indicadores de desempenho propostos pela International Water Association (IWA). Ademais, verificou-se a necessidade de uma melhoria no tratamento dos dados coletados. Atualmente, esses dados são fornecidos pelas companhias e, eventualmente, as informações são atribuídas sem critérios técnicos.

\section{Referências}

ALEGRE, H. et al. Controlo de Perdas de Água em Sistemas Públicos de Adução e Distribuição. Lisboa: Ed. IRAR, 2005. Série Guias Técnicos 3.

ALEGRE, H. et al. Indicadores de Desempenho Para Serviços de Abastecimento de Água. Lisboa: LNEC, 2004. Série Guias Técnicos.
ALEGRE, H. et al. Performance Indicators For Water Supply Services. $2^{\text {nd }}$. ed. Londres: IWA Publishing, 2006.

\section{ALEGRE, H.; COVAS, D. Gestão Patrimonial} de Infra-Estruturas de Abastecimento de Água: uma abordagem centrada na reabilitação. Lisboa, 2010. Séries Guias Técnicos, 16.

BARRETO, D. Perfil do Consumo Residencial e Usos Finais da água. Ambiente Construído, Porto Alegre, v. 8, n. 2, p. 23-40, abr./jun. 2008.

BARTH, F. T. Aspectos Institucionais do Gerenciamento de Recursos Hídricos. In: REBOUÇAS, A. da C. Águas Doces do Brasil: Capital Ecológico e Conservação. 2. ed. São Paulo: Ed. Escrituras, 2002.

BERESKIE, T. et al. Small Drinking Water Systems Under Spatiotemporal Water Quality Variability: a risk-based performance benchmarking framework. Environmental Monitoring and Assessment, v. 189, n. 9, p. 464, 2017.

BRITTO, A. L. (Coord.). Panorama do Saneamento Básico no Brasil: avaliação políticoinstitucional do setor de saneamento básico. Volume IV. Ministério das Cidades. Secretaria Nacional de Saneamento Ambiental, 2011.

GANJIDOOST, A. et al. Benchmark Performance Indicators for Utility Water and Wastewater Pipelines Infrastructure. Journal of Water Resources Planning and Management, v. 144, n. 3, p. 04018003, 2018. 
HAIDER, H.; SADIQ, R.; TESFAMARIAM, S. Inter-Utility Performance Benchmarking Model for Small-to-Medium-Sized Water Utilities: aggregated performance indices. Journal of Water Resources Planning and Management, v. 142, n. 1, p. 04015039, 2015.

MARENGO, J. A. Mudanças Climáticas Globais e Seus Efeitos Sobre a Biodiversidade: caracterização do clima atual e definição das alterações climáticas para o território brasileiro ao longo do século XXI. Brasília: Ministério do Meio Ambiente, 2007.

MOLINARI, A. Panorama Mundial. In: GALVÃO JUNIOR, A. de C.; SILVA. A. C. da (Orgs.). Regulação: indicadores para a prestação de serviços de água e esgoto. Fortaleza: Expressão Gráfica e Editora Ltda., 2006.

MOLINOS-SENANTE, M. et al. Assessing the Sustainability of Water Companies: a synthetic indicator approach. Ecological Indicators, v. 61, p. 577-587, 2016.

MORSCH, M. R. S.; MASCARÓ, J. J.; PANDOLFO, A. Sustentabilidade Urbana: recuperação dos rios como um dos princípios da infraestrutura verde. Ambiente Construído, Porto Alegre, v. 17, n. 4, p. 305-321, out./dez. 2017.
OFFICE OF WATER SERVICES. Annual Report and Accounts 2010-2011. Birmingham: Office of Water Services, 2011.

PERTEL, M.; AZEVEDO, J. P. S. de; VOLSCHAN JUNIOR, I. Uso de Indicadores de Perdas para Seleção de Um Benchmarking Entre as Companhias Estaduais de Serviço de Distribuição de Água no BRASIL. Engenharia Sanitária e Ambiental, v. 21, n. 1, p. 159-168, 2016.

SILVA, R. T. Aspectos Conceituais e Teóricos. In: GALVÃO JUNIOR, A. de C.; SILVA, A. C. da. Regulação: indicadores para a prestação de serviços de água e esgoto. Fortaleza: Expressão Gráfica e Editora Ltda., 2006.

SISTEMA NACIONAL DE INFORMAÇÕES SOBRE SANEAMENTO. Diagnóstico dos Serviços de Água e Esgotos (anos-base 2012 a 2018). Brasília: Ministério das Cidades. Disponível em: <http://www.snis.gov.br>. Acesso em: 30 abr. 2018.

VILANOVA, M. R. N.; MAGALHÃES FILHO, P.; BALESTIERI, J. A. P. Performance Measurement and Indicators for Water Supply Management: review and international cases. Renewable and Sustainable Energy Reviews, v. 43, p. 1-12, 2015.

\section{Saulo de Tarso Marques Bezerra}

Núcleo de Tecnologia, Campus Agreste | Universidade Federal de Pernambuco | Avenida Campina Grande, S/ N, Bloco K, Sala 6 | Caruaru - PE - Brasil | CEP 55014-900 | Tel.: (81) 2126-7383 | E-mail: s.bezerra@yahoo.com.br

\section{Monica Pertel}

Departamento de Recursos Hídricos e Meio Ambiente, Escola Politécnica | Universidade Federal do Rio de J aneiro | Centro de Tecnologia, Cidade Universitária, Bloco D, Sala 2024, Ilha do Fundão | Rio de J aneiro - RJ - Brasil | CEP 21941-909 | Tel.: (21) 2562-7982 | E-mail: monicapertel@poli.ufrj.br

\section{J osé Eloim Silva de Macêdo}

Centro Universitário Maurício de Nassau | BR 104, Km 68, S/ N, Bairro Agamenon Magalhães | Caruaru - PE - Brasil | CEP 55000-000 | Tel.: (81) 99677-0645 | E-mail: eloim_sm@hotmail.com

\section{Revista Ambiente Construído}

Associação Nacional de Tecnologia do Ambiente Construído

Av. Osvaldo Aranha, 99 - 3o andar, Centro

Porto Alegre - RS - Brasil

CEP $90035-190$

Telefone: +55 (51) 3308-4084

Fax: +55 (51) 3308-4054

www. seer. ufrgs. br/ ambienteconstruido

E-mail: ambienteconstruido@ufrgs.br 